\title{
INCISIONES VALVULADAS SIN SUTURA: DE LA CIRUGÍA DE CATARATA A LA CIRUGÍA VITREORRETINIANA
}

\section{SUTURELESS VALVED INCISIONS: FROM CATARACT TO VITREORETINAL SURGERY}

\author{
LÓPEZ-GUAJARDO L ${ }^{1}$, GONZÁLEZ-DEL-VALLE F ${ }^{2}$, TEUS-GUEZALA M ${ }^{3}$
}

Tras el establecimiento de la facoemulsificación como técnica quirúrgica de elección en la cirugía de la catarata, la incisión corneal valvulada (también llamada tunelizada) — unida a la aparición de lentes intraoculares (LIO) plegables (menor tamaño incisional) - permitió el cierre sin suturas de la incisión mediante un mecanismo de aposición de los labios de la herida favorecido por la presión intraocular. Desde su descripción, la incisión corneal valvulada ha ido ganando en aceptación, y así, en encuestas realizadas entre miembros de la ASCRS unos años después (2001), casi un 60\% de los oftalmólogos la describían como su técnica de elección. Este elemento supuso un importe avance quirúrgico, pues eliminó el astigmatismo inducido por la sutura, además de acelerar la recuperación al eliminar la posible reacción inflamatoria a elementos extraños en el tejido.

En la actualidad la idea de la incisión valvulada está siendo trasladado a las incisiones esclerales a nivel de pars plana para la realización de diversos procedimientos en cirugía vitreorretiniana. Así, conceptos como incisión oblicua, angulada, tunelizada. etc, que parecían reservados para la cirugía de polo anterior aparecen cada vez más frecuentemente en las publicaciones referidas a retina.

Tradicionalmente la vitrectomía de 20 gauges se ha realizado a través de esclerotomías con trayectoria directa (perpendicular a esclera), y, aunque, aparecieron diversos intentos de utilizar técnicas tunelizadas (1), estos no llegaron a establecerse de forma generalizada.

Con la aparición de la vitrectomía microincisional (25 gauges y 23 gauges) el menor diámetro de la incisión permitió plantearse la posibilidad de no utilizar suturas. La eliminación de la necesidad de suturas disminuye la reacción inflamatoria que encontramos en el postoperatorio de las vitrectomías a nivel de esclerotomía producida por el propio material de sutura. Estudios de topografía corneal han demostrado un menor astigmatismo postquirúrgico inducido en la vitrectomía microincisional sin suturas. Estos dos elementos aceleran el proceso de recuperación postoperatoria en nuestros enfermos. No obstante, no todo son ventajas en la vitrectomía microincisional sin suturas. Existe una mayor incidencia de hipotonía postoperatoria y, recientes estudios (2) han encontrado una mayor incidencia de endoftalmitis en vitrectomía de 25 gauges al compararla con 20 gauges. Ello debe hacer pensar que la incisión sin suturas no es un fin en si mismo y el cirujano debería suturar esta en caso de duda sobre su capacidad de cierre.

En un intento por mejorar la capacidad de cierre de las esclerotomías sin sutura en la vitrectomía microincisional se ha descrito la utilización de incisiones esclerales valvuladas. Así, aunque inicialmente la técnica de 25 gauges se describió por Fuji utilizando una incisión directa (perpendicular), posteriormente se ha propuesto su realización a través de incisiones oblicuas $(3,4)$, pues aportan una mayor capacidad de cierre (5) disminuyendo pérdida de líquido intraocular por reflujo. Debido a su mayor diámetro, la vitrectomía realizada a través de incisiones de 23 gauges ya fue descrita por Eckardt desde el principio utilizando incisiones anguladas.

Esta tendencia a la realización de incisiones esclerales oblicuas también ha sido utilizada para la

\footnotetext{
1 Doctor en Medicina. Hospital Príncipe de Asturias. Alcalá de Henares. Madrid. España. E-mail: 1lguajardo@icam.es

2 Licenciado en Medicina. Hospital La Mancha Centro. Alcázar de San Juan. Ciudad Real. España.

3 Doctor en Medicina. Hospital Príncipe de Asturias. Universidad de Alcalá. Alcalá de Henares. Madrid. España.
} 
administración intravítrea de fármacos mediante inyección vía pars plana. Así, se han descrito inyecciones esclerales oblicuas, tanto en dos planos (6) como uno (7), en un intento de disminuir la pérdida de fármaco que se produce por reflujo y posterior acumulo subconjuntival (ampolla) en el caso de la inyección directa (perpendicular) a esclera.

Otro procedimiento realizado habitualmente por el cirujano de retina, la colocación de lentes intraoculares (LIO) en sulcus en ausencia de soporte capsular normalmente precisa del concurso de suturas para su fijación. Las incisiones esclerales tunelizadas también pueden ser utilizadas en este tipo de casos eliminando la necesidad de suturas. Gabor (8) ha descrito una técnica de atrapamiento de los hápticos en incisiones esclerales tunelizadas paralelas a limbo construidas a $1,5 \mathrm{~mm}$ de este. En nuestra experiencia, el atrapamiento del háptico en el túnel escleral permite buen centrado y ausencia de inclinación del implante. La no utilización de suturas elimina la posibilidad de erosión tardía de la sutura y posterior luxación del implante.

Así pues, la utilización de incisiones esclerales valvuladas/oblicuas/tunelizadas en un intento de mejorar la capacidad de cierre de la herida quirúrgica sin sutura parece ser una opción a considerar al plantearse determinados procedimientos que requieran acceso a la cavidad vítrea. Conforme se vaya acumulando mayor experiencia, podrá determinarse la importancia real de esta aproximación y puede que, a semejanza de lo ocurrido con la inci- sión corneal valvulada en cirugía de catarata, llegue a ser una técnica ampliamente utilizada por un importante número de cirujanos.

\section{BIBLIOGRAFÍA}

1. Chen JC. Sutureless pars plana vitrectomy through selfsealing sclerotomies. Arch Ophthalmol 1996; 114: 12731275.

2. Kunimoto DY, Kaiser RS. Incidence of endophthalmitis after 20- and 25-gauge vitrectomy. Ophthalmology 2007; 114: 2133-2137.

3. López-Guajardo L, Pareja-Esteban J, Teus-Guezala MA. Oblique sclerotomy technique for prevention of incompetent wound closure in transconjunctival 25-gauge vitrectomy. Am J Ophthalmol 2006; 141: 1154-1156.

4. Shimada H, Nakashizuka H, Mori R, Mizutani Y, Hattori T. 25-gauge scleral tunnel transconjunctival vitrectomy. Am J Ophthalmol 2006; 142: 871-873.

5. López-Guajardo L, Vleming-Pinilla E, Pareja-Esteban J, Teus-Guezala, M. Ultrasound biomicroscopy study of direct and oblique 25-gauge vitrectomy sclerotomies. Am J Ophthalmol 2007; 143: 881-883.

6. Rodrigues EB, Meyer CH, Grumann A Jr, Shiroma $H$, Aguni JS, Farah ME. Tunneled scleral incision to prevent vitreal reflux after intravitreal injection. Am J Ophthalmol 2007; 143: 1035-1037.

7. Lopez-Guajardo L, Del Valle FG, Moreno JP, Teus M A. Reduction of pegaptanib loss during intravitreal delivery using an oblique injection technique. Eye 2008 [Epub ahead of print $]$.

8. Gabor SG, Pavlidis MM. Sutureless intrascleral posterior chamber intraocular lens fixation. J Cataract Refract Surg 2007; 33: 1851-1854. 